\title{
OPTIMAL CONTROL PROBLEMS GOVERNED BY AN ELLIPTIC DIFFERENTIAL EQUATION WITH CRITICAL EXPONENT
}

\author{
Gengsheng Wang
}

Department of Mathematics, Huazhong Normal University

\begin{abstract}
This work is concerned with analysis and optimal control of a semilinear elliptic partial differential equation involving critical exponent. Some necessary conditions for optimality are given.
\end{abstract}

Key words and phrases: Minimal positive solution, state constraint, finite codimensionality, Penalty functional.

\section{INTRODUCTION}

We discuss the optimal control problem for which the state is governed by a semilinear elliptic partial differential equation with a distributed control.

The system reads

$$
\left\{\begin{array}{l}
-\Delta y=y^{p}+u, \quad \text { in } \Omega \\
\left.y\right|_{\partial \Omega}=0
\end{array}\right.
$$

Where $\Omega \subset R^{N}$ (with $N \geq 3$ ) is a bounded region with $\partial \Omega$ smooth. $p=\frac{N+2}{N-2}$ is the critical sobolev exponent.

The cost functional is given by

$$
L(y, u)=G(y)+H(u)
$$

We assume that

$\left(\mathbf{H}_{\mathbf{L}}\right) \quad G$ and $H: L^{2}(\Omega) \longrightarrow \bar{R}=(-\infty,+\infty]$ are proper, convex and lower semicontinuous.

In section 2, we will see that there is a minimal positive

solution $y(x ; u) \in H_{0}^{1}(\Omega)$ for each $u \in B_{r}^{+}(0) \subset L^{\infty}(\Omega) \subset L^{2}(\Omega)$, where $B_{r}^{+}(0)$ is given by

$$
B_{r}^{+}(0)=\left\{u \in L^{\infty}(\Omega) \mid\|u\|_{\infty} \leq r \quad \text { and } u(x) \geq 0, \quad \text { a.e. } \quad x \in \Omega\right\}
$$

The original version of this chapter was revised: The copyright line was incorrect. This has been corrected. The Erratum to this chapter is available at DOI: 10.1007/978-0-387-35359-3_40 
and $r>0$ is a constant given in $\S 2$.

Note that here we define $y(x ; 0) \equiv 0$. Thus we may consider $u \in B_{r}^{+}(0)$ as the control and $y(x ; u)$, the minimal positive solution of $(1.1)$ corresponding to the state.

We assume

$\left(\mathbf{H}_{\mathbf{F}}\right) \quad$ Let $Y$ be a Banach space with strict convex dual $Y^{*}, F: H_{0}^{1}(\Omega) \rightarrow Y$ be continuously Frechet differentiable and $Q \subset Y$ be a closed and convex subset.

Set

$$
\begin{gathered}
A=\left\{(y(x ; u), u) \in L^{2}(\Omega) \times L^{2}(\Omega) \mid u \in B_{r}^{+}(0), y(x ; u)\right. \text { is the } \\
\text { minimal positive solution of }(1.1) \\
\text { corresponding to } u \text { and } y(x ; 0) \equiv 0\}
\end{gathered}
$$

A pair $(y, u) \in A$ is called a feasible pair.

$$
A_{a d}=\{(y(x ; u), u) \in A \mid F(y(x ; u)) \subset Q\}
$$

A pair $(y, u) \in A_{a d}$ is called an admissible pair.

Note that $F(y) \subset Q$ is a kind of state constraint which was given by X.Li and J.Yong(cf.[3]). For its applications, we refer readers to [2] and [3].

We formulate the optimal control problem as follows

(P) $\quad$ Inf $L(y, u) \quad$ over all $(y, u) \in A_{a d}$

We shall study the necessary conditions for the problem $(P)$ in this paper.

\section{THE MINIMAL POSITIVE SOLUTION}

We first quote a result of [5] as follows.

Theorem A: For any $u \in H^{-1}(\Omega)$ with $\|u\|_{H^{-1}} \leq C_{N} S^{\frac{N}{2}}$, problem (2.1) possesses at least one positive solution $y$ with $y \not \equiv 0$ in $\Omega$. Where $C_{N}=$ $\frac{4}{N-2}\left(\frac{N-2}{N+2}\right)^{\frac{N+2}{4}}$ and $S$ is the best sobolev constant for the embedding $H_{0}^{1}(\Omega) \rightarrow$ $L^{p}(\Omega)$.

From Theorem A and the methods of monotone interation we can prove the existence of minimal positive solution for problem (2.1).

Theorem 2.1 Under the assumption of Theorem A, Problem (2.1) possesses a unique minimal positive solution $y \in C^{\alpha}(\Omega)$ for some $\alpha \in(0,1)$ if $u \in L^{\infty}(\Omega)$.

In the following we discuss some properties of the minimal positive solution of (1.1).

Lemma 2.1 Let $y(x ; u)$ be the minimal positive solution of (1.1), then the corresponding eigenvalue problem

$$
\left\{\begin{array}{l}
-\Delta \varphi=\lambda p[y(x ; u)]^{p-1} \varphi, \quad \text { in } \quad \Omega \\
\varphi \in H_{0}^{1}(\Omega)
\end{array}\right.
$$


has the first eigenvalue $\lambda_{1}(u)>1$ for all $u \in B_{R}^{+}(0)$ and the corresponding eigenfunction $\varphi_{1}>0$ in $\Omega$.

Where

$$
B_{R}^{+}=\left\{u \in H_{0}^{-1}(\Omega) \mid\|u\|_{H_{0}^{1}(\Omega)} \leq R, u \geq 0 \text { in } \Omega\right\}
$$

and $R<C_{N} S^{\frac{N}{2}}$.

Proof: By the standard argument we can prove that the minimum

$$
\lambda_{1}=\operatorname{Inf}\left\{\int_{\Omega}|\nabla v|^{2} d x \mid v \in H_{0}^{1}(\Omega), \int_{\Omega} p y^{p-1}(x ; u) v^{2} d x=1\right\}
$$

can be achieved by some function $\varphi_{1}>0$. Thus eigenvalue problem (2.2) has a solution $\left(\lambda_{1}, \varphi_{1}\right)$. Now we prove that $\lambda_{1}>1$.

Indeed, for any $u \in B_{R}^{+}(0)$, we can find a function $w \in H_{0}^{-1}(\Omega)$ with $\|w\|_{H_{0}^{-1}} \leq C_{N} S^{\frac{N}{2}}, w \geq u, w \not \equiv u$ a.e. in $\Omega$ such that problem (2.1) (corresponding to $w)$ possesses a minimal positive solution $y(x ; w)$. Let $y(x ; u)$ be the solution of $(2.1)$, we have

$$
\lambda_{1} \int_{\Omega} p y^{p-1}(x ; u) \varphi_{1}[y(x ; w)-y(x: u)] d x \int_{\Omega} p y^{p-1}(x ; u) \varphi_{1}(y(x ; w)-y(x ; u)) d x
$$

Which gives $\lambda_{1}>1$ for all $u \in B_{R}^{+}(0)$. This completes the proof.

Theorem 2.2 Assume $u \in B_{R}^{+}(0)$ and $y(x ; u)$ be a minimal positive solution of (1.1) corresponding to $u$. Then for any $g(x) \in H_{0}^{-1}(\Omega)$, The problem

$$
\left\{\begin{array}{l}
-\Delta \omega=p y^{p-1}(x ; u) \omega+g(x) \\
\omega \in H_{0}^{1}(\Omega)
\end{array}\right.
$$

has a unique solution $\omega$ satisfying

$$
\|\omega\|_{H_{0}^{1}(\Omega)} \leq C \mid g \|_{H_{0}^{-1}(\Omega)}
$$

for some constant $C>0$.

Proof: By a standard argument and Lemma 2.1, one can get the existence of the solution of the equation (2.4).

Now we are on the position to prove(2.5).

Let $\omega$ be the solution of (2.5). Multiplying (2.5) by $\omega$ and integrating by parts we have

$$
\int_{\Omega}|\nabla \omega|^{2} d x=\int_{\Omega} p y^{p-1}(x ; u) \omega^{2} d x+\int_{\Omega} g \omega d x .
$$

Now Lemma 2.1 implies 


$$
\begin{gathered}
\left(1-\frac{1}{\lambda_{1}}\right)\|\omega\|_{H_{0}^{1}(\Omega)}^{2} \leq C\|g\|_{H_{0}^{-1}(\Omega)}\|\omega\|_{H_{0}^{1}(\Omega)} \\
\leq \epsilon\|\omega\|_{H_{0}^{1}(\Omega)}^{2}+C_{\epsilon}\|g\|_{H_{0}^{-1}(\Omega)}^{2}
\end{gathered}
$$

From Lemma2.1 we can choose $\epsilon$ small enough so that $\left(1-\frac{1}{\lambda_{1}}-\epsilon\right) \geq \lambda_{2}>0$ for some constant $\lambda_{2}>0$.

Thus

$$
\|\omega\|_{H_{0}^{1}(\Omega)} \leq \frac{C_{\epsilon}}{\lambda_{2}}\|g\|_{H_{0}^{-1}(\Omega)}
$$

This gives (2.5) by taking $C=\frac{C_{\epsilon}}{\lambda_{2}}$.

The uniqueness of the solution for (2.4) comes from (2.5).

Corollary 2.1 Let $u \in B_{R}^{+}(0)$ and $y(x ; u)$ be the minimal solution of (1.1). Then $y(x, u)$ is continuous in $H_{0}^{-1}(\Omega)$ with respect to control function $u$.

Proof: Define

$$
\begin{gathered}
F: H_{0}^{-1}(\Omega) \times H_{0}^{1}(\Omega) \rightarrow H_{0}^{-1}(\Omega) \text { by } \\
F(u, y)=\Delta y+y^{p}+u, \text { for }(u, y) \in H_{0}^{-1}(\Omega) \times H_{0}^{1}(\Omega)
\end{gathered}
$$

From Lemma 2.1 and Theorem 2.2, we know that

$$
F_{y}(u, y) \omega=\Delta \omega+p y^{p-1}+p y^{p-1}(x ; u) \omega
$$

is an isomorphism of $H_{0}^{1}(\Omega)$ onto $H_{0}^{-1}(\Omega)$.

It follows from Implicit Function Theorem that the solution of $F(u, y)=0$ near $(u, y(x ; u))$ is given by a continuous curve.

Theorem 2.3 Let $u, v \in B_{R}^{+}(0)$ and $y(x, u), y(x, v)$ be the minimal positive solution of (1.1) corresponding to $u, v$ respectively. If $u \rightarrow v$ in $H_{0}^{-1}(\Omega)$ and $u-v$ dosen't change the sign. Then

$$
\|y(x ; u)-y(x ; v)\|_{H_{0}^{1}(\Omega)} \leq C\|u-v\|_{H_{0}^{-1}(\Omega)}
$$

for $\|u-v\|_{H_{0}^{1}(\Omega)}$ small enough.

Where $C$ is a constant independent of $u$.

Proof: Without loss of generality, we may assume that $u \geq v$, a,e. in $\Omega$.

By Remark 2.1 and (1.1) we have

$$
\begin{aligned}
& \int_{\Omega}|\nabla(y(x ; u)-y(x ; v))|^{2} d x \\
& \leq p \int_{\Omega} y^{p-1}(x ; u)(y(x ; u)-y(x ; v))^{2} d x+\int_{\Omega}(u-v)(y(x ; u)-y(x ; v)) d x
\end{aligned}
$$

By lemma 2.1, Holder's inequality and Young's inequality, we have

$$
\begin{aligned}
& \left(1-\frac{1}{\lambda_{1}(u)}\right) \int_{\Omega}|\nabla(y(x ; u)-y(x ; v))|^{2} d x \\
& \leq \epsilon\|y(x ; u)-y(x ; v)\|_{H_{0}^{1}(\Omega)}^{2}+C_{\epsilon}\|u-v\|_{H_{0}^{-1}(\Omega)}^{2}
\end{aligned}
$$


for any $\epsilon>0$. Where $C_{\epsilon}$ is a positive constant depending on $\epsilon$.

Note that $\lambda_{1}(u)$ is the first eigenvalue for the problem (2.2) corresponding to $y(x ; u)$. By corollary 2.1, as $u \rightarrow v$ in $H_{0}^{-1}(\Omega), y(x ; u) \rightarrow y(x ; v)$ in $H_{0}^{1}(\Omega)$. Then by (2.3), $\lambda_{1}(u) \rightarrow \lambda_{1}(v)$.

Thus, as $\|u-v\|_{H_{0}^{-1}(\Omega)}$ small enough, we have

$$
\left(1-\frac{1}{\lambda_{1}(v)}-\epsilon_{1}-\epsilon\right)\|y(x ; u)-y(x ; v)\|_{H_{0}^{1}(\Omega)} \leq C_{\epsilon}\|u-v\|_{H_{0}^{-1}(\Omega)}
$$

for some $\epsilon_{1}>0$ with $1-\frac{1}{\lambda_{1}(v)}-\epsilon_{1}>0$.

This completes the proof.

\section{FINITE CODIMENSIONALITY}

In this section, we will give some results relative to finite codimensionality of a set. For the detail, we refer readers to [3] and [6].

Lemma 3.1 Let $Q_{1}$ and $Q_{2}$ be subsets of some Banach space $X$. Let $Q_{1}$ be finite codimensional in $X$. Then for any $\alpha \in R \backslash\{0\}, \beta \in R$,

$$
\alpha Q_{1}-\beta Q_{2} \equiv\left\{\alpha x_{1}-\beta x_{2} \mid x_{1} \in Q_{1}, x_{2} \in Q_{2}\right\}
$$

is finite codimensional in $X$.

Lemma 3.2 Let $Q$ be finite codimensional in $X$. Let $\left\{f_{n}\right\}_{n \geq 1} \subset X^{*}$ with $\left|f_{n}\right| \geq \delta>0, f_{n} \rightarrow f \in X^{*}$ in the weak-star topology, and

$$
\left\langle f_{n}, x\right\rangle \geq-\epsilon_{n}, \quad \forall x \in Q, \quad n \geq 1
$$

when $\epsilon_{n} \rightarrow 0$. Then $f \neq 0$.

\section{NECESSARY CONDITION FOR OPTIMALITY}

In this section, we discuss the necessary conditions for $\left(y^{*}, u^{*}\right)$ to be an optimal pair for $(P)$.

Our basic assumptions are given by $\left(H_{L}\right)$ and $\left(H_{F}\right)$ in $\S 1$.

Let $\left(y^{*}, u^{*}\right)$ be optimal for the problem $(P)$.

Consider the variational systems of (1.1) as follows:

$$
\left\{\begin{array}{l}
-\Delta z=p y^{*(p-1)} z+\left(v-u^{*}\right)^{+}, \quad x \in \Omega \\
\left.z\right|_{\partial \Omega}=0
\end{array}\right.
$$

and

$$
\left\{\begin{array}{l}
-\Delta z=p y^{*(p-1)} z+\left(v-u^{*}\right)^{-}, \quad x \in \Omega \\
\left.z\right|_{\partial \Omega}=0
\end{array}\right.
$$

By Theorem 2.2, for each $v \in B_{r}^{+}(0)$, both (4.1) and (4.2) have a unique solution $z\left(. ; v^{+}\right)$and $z\left(. ; v^{-}\right)$in $H_{0}^{1}(\Omega)$. 
Thus we may define

$R^{+}=\left\{z\left(. ; v^{+}\right) \mid v \in B_{r}^{+}(0), z\left(. ; v^{+}\right)\right.$is the solution of (4.1) corresponding to $\left.v\right\}$ $R^{-}=\left\{z\left(\cdot ; v^{-}\right) \mid v \in B_{r}^{+}(0), z\left(\cdot ; v^{-}\right)\right.$is the solution of $(4.2)$ corresponding to $\left.v\right\}$

Our another basic assumption which plays a key role in dealing with the state constraint is as follows:

$\left(H_{R}\right)$ Both $F^{\prime}\left(y^{*}\right) R^{+}-Q$ and $F^{\prime}\left(y^{*}\right) R^{-}-Q$ have finite codimentionality.

The following results is important for us to introduce our penalty functionals in the proof of our main Theorem 4.1.

Lemma 4.1 Let $H$ be a Hilbert space, $f: H \rightarrow \bar{R}$ be proper convex and lower semicontinuous. Suppose that $\partial f$ (the subdifferential of $f$ ) is locally bounded at $y^{*}$. Then there exists a neighborhood $O\left(y^{*}\right)$ of $y^{*}$ such that $f_{\lambda}(y) \rightarrow$ $f(y)$ uniformly in $O\left(y^{*}\right)$, where $f_{\lambda}$ is the regularization of $f$ (cf. [4], [7]).

Proof: It is trivial from [4] and [7].

Our main results on the necessary condition for $\left(y^{*}, u^{*}\right)$ to be an optimal pair are as follows:

Theorem 4.1 Let $\left(y^{*}, u^{*}\right)$ be an optimal pair for $\left.P\right)$ and $\left(H_{L}\right),\left(H_{F}\right)$ and $\left(H_{R}\right)$ hold. Assume that $\partial G$ and $\partial H$, the subdifferentials of $G$ and $H$ are locally bounded at $y^{*}$ and $u^{*}\left(\right.$ in $\left.L^{2}(\Omega)\right)$ respectively. Then there exists a triplet

$$
\begin{gathered}
(\lambda, \psi, q) \in[-1,0] \times H_{0}^{1}(\Omega) \times Y^{*}, \quad \text { such that }(\lambda, q) \neq 0, \\
\left\langle q, \eta-F\left(y^{*}\right)\right\rangle \leq 0, \quad \forall \eta \in Q . \\
\left\{\begin{array}{l}
-\Delta \Psi=p y^{*(p-1)} \Psi+\lambda \alpha-\left[F^{\prime}\left(y^{*}\right)\right]^{*} q \quad \text { in } \Omega \\
\left.\Psi\right|_{\partial \Omega}=0,
\end{array}\right.
\end{gathered}
$$

where $\alpha \in \partial G\left(y^{*}\right)$

and

$$
\left\langle\Psi+\lambda \beta, v-u^{*}\right\rangle \leq 0 \quad \text { for any } \quad v \in B_{r}^{+}(0)
$$

Where $\beta \in \partial H\left(u^{*}\right)$.

In the case $N\left[F^{\prime}\left(y^{*}\right)\right]^{*}=0$ (i.e. $\left[F^{\prime}\left(y^{*}\right)\right]^{*}$ is injective), $(\lambda, \psi) \neq 0$.

Proof: Without lose of generality, assume that

$$
L\left(y^{*}, u^{*}\right)=G\left(y^{*}\right)+H\left(u^{*}\right)=0 .
$$

Since $\partial G$ and $\partial H$ are locally bounded at $y^{*}$ and $u^{*}$ respectively, by lemma 4.1 , we obtain that there exist neighborhoods $O\left(y^{*}\right)$ and $O\left(u^{*}\right)$ of $y^{*}$ and $u^{*}$ in $L^{2}(\Omega)$ respectively, such that

$$
\begin{aligned}
& G(y)+H(u) \geq G_{\lambda}(y)+H_{\lambda}(u) \rightarrow G(y)+H(u) \\
& \text { as } \lambda \rightarrow 0 \text { uniformly in } y \in O\left(y^{*}\right) \text { and } u \in O\left(y^{*}\right) .
\end{aligned}
$$


Thus for each $\epsilon>0$, there exists a $\delta(\epsilon)>0(\delta(\epsilon) \rightarrow 0$ as $\epsilon \rightarrow 0)$, such that

$$
G(y)+H(u)+\epsilon \geq G_{\delta(\epsilon)}(y)+H_{\delta(\epsilon)}(u)+\epsilon>G(y)+H(u)
$$

for all $(y, u) \in O\left(y^{*}\right) \times O\left(u^{*}\right)$.

Let $U=\left(B_{r}^{+}(0), d\right)$ with $d(u, v)=\|u-v\|$, where the norm is taken in $L^{2}(\Omega)$. Then $U$ is a metric space. Note that $B_{r}^{+}(0) \subset L^{\infty}(\Omega) \subset L^{2}(\Omega)$ and one can check easily that $B_{r}^{+}(0)$ is closed in $L^{2}(\Omega)$. Thus $U$ is a complete metric space.

Now we define $L_{\epsilon}: U \rightarrow R$ by

$$
L_{\epsilon}(u)=\left\{d_{Q}^{2}(F(y(u)))+\left[G_{\delta(\epsilon)}(y(u))+H_{\delta(\epsilon)}(u)+\epsilon\right]^{2}\right\}^{\frac{1}{2}} .
$$

Where $d_{Q}(w)=\operatorname{Inf}\|w-z\|, z \in Q$ and $y \equiv y(x ; u)$ is the unique minimal positive solution of (1.1) corresponding to $u$.

By Ekeland's variational principle, there exists a $u^{\epsilon} \in U$ for each $\epsilon>0$ such that

$$
d\left(u^{*}, u^{\epsilon}\right) \leq \sqrt{\epsilon}, \quad \text { i.e. : } \quad\left\|u^{*}-u^{\epsilon}\right\| \leq \sqrt{\epsilon}
$$

and

$$
L_{\epsilon}(u)-L_{\epsilon}\left(u^{\epsilon}\right) \geq-\sqrt{\epsilon} d\left(u, u^{\epsilon}\right), \quad \forall u \in U .
$$

Let $v \in U$, we define

$$
u_{\rho}^{\epsilon}=u^{\epsilon}+\rho\left(v-u^{\epsilon}\right)^{+} .
$$

It's clear that $u_{\rho}^{\epsilon} \in U$ and

$$
u_{\rho}^{\epsilon}-u^{\epsilon}=\rho\left(v-u^{\epsilon}\right)^{+} \rightarrow 0 \quad \text { in } \quad \mathrm{L}^{\infty}(\Omega) \quad \text { as } \quad \rho \rightarrow 0^{+} .
$$

Let $y_{\rho}^{\epsilon} \equiv y_{\rho}^{\epsilon}\left(\cdot ; u_{\rho}^{\epsilon}\right)$ be the minimal positive solution of (1.1) corresponding to $u_{\rho}^{\epsilon}$ and $z_{\rho}^{\epsilon} \equiv \frac{y_{\rho}^{\epsilon}-y^{\epsilon}}{\rho}$.

Consider

$$
\left\{\begin{array}{l}
-\Delta z^{\epsilon}=p\left(y^{\epsilon}\right)^{p-1} z^{\epsilon}+\left(v-u^{\epsilon}\right)^{+}, \quad x \in \Omega \\
\left.z^{\epsilon}\right|_{\partial \Omega}=0
\end{array}\right.
$$

We have

$$
-\Delta\left(z^{\epsilon}-z_{\rho}^{\epsilon}\right)-p\left(y_{\epsilon}\right)^{p-1}\left(z^{\epsilon}-z_{\rho}^{\epsilon}\right)=\left(p\left(y^{\epsilon}\right)^{p-1}-a_{\rho}^{\epsilon}\right) z_{\rho}^{\epsilon},
$$

where $a_{\rho}^{\epsilon}=\int_{0}^{1} p\left[y^{\epsilon}+t\left(y_{\rho}^{\epsilon}-y^{\epsilon}\right)\right]^{p-1} d t$.

Multiplying (4.11) by $\left(z^{\epsilon}-z_{\rho}^{\epsilon}\right.$ ) and interating on $\Omega$, we obtain (note that both $y^{\epsilon}$ and $y_{\rho}^{\epsilon}$ are positive)

$$
\begin{aligned}
& \int_{\Omega}\left|\nabla\left(z^{\epsilon}-z_{\rho}^{\epsilon}\right)\right|^{2} d x-\int_{\Omega} p\left(y^{\epsilon}\right)^{p-1} \cdot\left(z^{\epsilon}-z_{\rho}^{\epsilon}\right)^{2} d x \\
& =\int_{\Omega}\left[p\left(y^{\epsilon}\right)^{p-1}-a_{\rho}^{\epsilon}\right] z_{\rho}^{\epsilon} \cdot\left(z_{\rho}^{\epsilon}-z^{\epsilon}\right) d x \\
& \leq \frac{C_{\epsilon}}{2}\left[\int_{\Omega}\left|y_{\rho}^{\epsilon}-y^{\epsilon}\right|^{\frac{N(p-1)}{2}} d x\right]^{\frac{4}{N}} \cdot\left\|z_{\rho}^{\epsilon}\right\|_{H_{0}^{1}}^{2}+\frac{\epsilon}{2}\left\|z^{\epsilon}-z_{\rho}^{\epsilon}\right\|_{H_{0}^{1}}^{2}
\end{aligned}
$$

for any $\epsilon>0$. 
By Lemma 2.1,

$$
\left(1-\frac{1}{\lambda_{1}}-\epsilon\right)\left\|z^{\epsilon}-z_{\rho}^{\epsilon}\right\|_{H_{0}^{1}}^{2} \leq \frac{C_{\epsilon}}{2}\left[\int_{\Omega}\left|y_{\rho}^{\epsilon}-y^{\epsilon}\right|^{\frac{N(p-1)}{2}} d x\right]^{\frac{4}{N}} \cdot\left\|z_{\rho}^{\epsilon}\right\|_{H_{0}^{1}}^{2}
$$

Taking $\epsilon$ small enough s.t. $1-\frac{1}{\lambda_{1}}-\epsilon \leq r>0$, we have

$$
\left\|z^{\epsilon}-z_{\rho}^{\epsilon}\right\|_{H_{0}^{1}}^{2} \leq \frac{1}{r} \cdot \frac{C}{2}\left[\int_{\Omega}\left|y_{\rho}^{\epsilon}-y^{\epsilon}\right|^{\frac{N(p-1)}{2}} d x\right]^{\frac{4}{N}} \cdot\left\|z_{\rho}^{\epsilon}\right\|_{H_{0}^{1}}^{2}
$$

Since $p=\frac{N+2}{N-2}$ and $y_{\rho}^{\epsilon} \rightarrow y^{\epsilon}$ in $H_{0}^{1}(\Omega)$, we have

$$
\left[\int_{\Omega}\left|y_{\rho}^{\epsilon}-y^{\epsilon}\right|^{\frac{N(p-1)}{2}}\right]^{\frac{4}{N}} \leq C_{1} \cdot\left\|y_{\rho}^{\epsilon}-y^{\epsilon}\right\|_{H_{0}^{1}}^{\frac{4}{N-2}}
$$

On the other hand, by Theorem 2.3,

$$
\left\|z_{\rho}^{\epsilon}\right\|_{H_{0}^{1}}=\left\|\frac{y_{\rho}^{\epsilon}-y^{\epsilon}}{\rho}\right\|_{H_{0}^{1}} \leq \frac{C_{2} \cdot \rho\left\|\left(v-u^{\epsilon}\right)^{+}\right\|_{L^{2}(\Omega)}}{\rho} \leq C_{3}
$$

Thus (4.12) gives us

$$
\left\|z^{\epsilon}-z_{\rho}^{\epsilon}\right\|_{H_{0}^{1}} \leq C_{4} \cdot\left\|y_{\rho}^{\epsilon}-y^{\epsilon}\right\|_{H_{0}^{1}}^{\frac{2}{N-2}} \rightarrow 0 \quad \text { as } \quad \rho \rightarrow 0 .
$$

Thus we obtain

$$
y_{\rho}^{\epsilon}=y^{\epsilon}+\rho z^{\epsilon}+\rho o(1) \text { in } H_{0}^{1}(\Omega)
$$

Next we estimate $G_{\delta(\epsilon)}\left(y_{\rho}^{\epsilon}\right)-G_{\delta(\epsilon)}\left(y^{\epsilon}\right)$ and $H_{\delta(\epsilon)}\left(u_{\rho}^{\epsilon}\right)-H_{\delta(\epsilon)}\left(u^{\epsilon}\right)$.

Clearly, we have

$$
\begin{aligned}
& \frac{G_{\delta(\epsilon)}\left(y_{\rho}^{\epsilon}\right)-G_{\delta(\epsilon)}\left(y^{\epsilon}\right)}{\rho}=\left\langle\dot{G}_{\delta(\epsilon)}\left(y^{\epsilon}\right), z_{\rho}^{\epsilon}\right\rangle+\frac{1}{\rho} o\left(\left\|y_{\rho}^{\epsilon}-y^{\epsilon}\right\|_{L^{2}}\right) \\
& =\left\langle\dot{G}_{\delta(\epsilon)}\left(y^{\epsilon}\right), z_{\rho}^{\epsilon}\right\rangle+o(1), \text { as } \rho \rightarrow 0
\end{aligned}
$$

and

$$
\frac{H_{\delta(\epsilon)}\left(u_{\rho}^{\epsilon}\right)-H_{\delta(\epsilon)}\left(u^{\epsilon}\right)}{\rho}=\left\langle\dot{H}_{\delta(\epsilon)}\left(u^{\epsilon}\right),\left(v-u^{\epsilon}\right)^{+}\right\rangle+o(1), \quad \text { as } \quad \rho \rightarrow 0
$$

We have

$$
\frac{L_{\epsilon}\left(u_{\rho}^{\epsilon}\right)-L_{\epsilon}\left(u^{\epsilon}\right)}{\rho} \geq-\sqrt{\epsilon}\left\|\left(v-u^{\epsilon}\right)^{+}\right\|_{L^{2}} \geq-\sqrt{\epsilon} M .
$$

It's clear that

$$
L_{\epsilon}\left(u_{\rho}^{\epsilon}\right)=L_{\epsilon}\left(u^{\epsilon}\right)+o(1) \quad \text { in } \quad L^{2}(\Omega) \text { as } \rho \rightarrow 0^{+} .
$$

One can check that $L_{\epsilon}\left(u^{\epsilon}\right) \neq 0$ for $\epsilon$ small enough. 
So we have

$$
\begin{aligned}
& -\sqrt{\epsilon} M \leq \frac{1}{L_{\epsilon}\left(u_{p}^{\epsilon}\right)+L_{\epsilon}\left(u^{\epsilon}\right)}\left\{\frac{\left[G_{\delta(\epsilon)}\left(y_{\rho}^{\epsilon}\right)+H_{\delta(\epsilon)}\left(u_{\rho}^{\epsilon}\right)+\epsilon\right]^{2}}{\rho}\right. \\
& \left.-\frac{\left[G_{\delta(\epsilon)}\left(y^{\epsilon}\right)+H_{\delta(\epsilon)}\left(u^{\epsilon}\right)+\epsilon\right]^{2}}{\rho}+\frac{d_{Q}^{2}\left(F\left(y_{\rho}^{\epsilon}\right)\right)-d_{Q}^{2}\left(F\left(y^{\epsilon}\right)\right)}{\rho}\right\} \\
& \rightarrow \frac{G_{\delta(\epsilon)}\left(y^{\epsilon}\right)+H_{\delta(\epsilon)}\left(u^{\epsilon}\right)+\epsilon}{L_{\epsilon}\left(u^{\epsilon}\right)}\left[\left\langle\dot{G}_{\delta(\epsilon)}\left(y^{\epsilon}\right), z^{\epsilon}\right\rangle+\left\langle\dot{H}_{\delta(\epsilon)}\left(u^{\epsilon}\right),\left(v-u^{\epsilon}\right)^{+}\right]\right. \\
& +\left\langle\frac{d_{Q}\left(F\left(y^{\epsilon}\right)\right) \xi^{\epsilon}}{L_{\epsilon}\left(u^{\epsilon}\right)}, F^{\prime}\left(y^{\epsilon}\right) z^{\epsilon}\right\rangle
\end{aligned}
$$

Define

$$
\lambda_{0}^{\epsilon}=\frac{G_{\delta(\epsilon)}\left(y^{\epsilon}\right)+H_{\delta(\epsilon)}\left(u^{\epsilon}\right)+\epsilon}{L_{\epsilon}\left(u^{\epsilon}\right)} \quad \in[0,1], \quad q^{\epsilon}=\frac{d_{Q}\left(F\left(y^{\epsilon}\right)\right) \xi^{\epsilon}}{L_{\epsilon}\left(u^{\epsilon}\right)}
$$

Then

$$
-\sqrt{\epsilon} M \leq \lambda_{0}^{\epsilon}\left[\left\langle\dot{G}_{\delta(\epsilon)}\left(y^{\epsilon}\right), z^{\epsilon}\right\rangle+\left\langle\dot{H}_{\delta(\epsilon)}\left(u^{\epsilon}\right),\left(v-u^{\epsilon}\right)^{+}\right\rangle\right]+\left\langle q^{\epsilon}, F^{\prime}\left(y^{\epsilon}\right) z^{\epsilon}\right\rangle
$$

Since $Y^{*}$ is strictly convex, we have

$$
\left|\lambda_{0}^{\epsilon}\right|^{2}+\left\|q^{\epsilon}\right\|_{Y^{*}}^{2}=1
$$

and

$$
\left\langle q^{\epsilon}, \eta-F\left(y^{\epsilon}\right)\right\rangle \leq 0 \quad \forall \eta \in Q
$$

In order to pass to the limits for $\epsilon \rightarrow 0^{+}$,

We first consider equation as follows:

$$
\left\{\begin{array}{l}
-\Delta z=p\left(y^{*}\right)^{p-1} z+\left(v-u^{*}\right)^{+}, \quad x \in \Omega \\
\left.z\right|_{\partial \Omega}=0
\end{array}\right.
$$

which has a unique solution $z$ in $H_{0}^{1}(\Omega)$, by Theorem 2.2 .

By the similar arguments in (4.12) and (4.13), We have

$$
z^{\epsilon} \rightarrow z \operatorname{in} H_{0}^{1}(\Omega)
$$

Consider $\left\{\dot{G}_{\delta(\epsilon)}\left(y^{\epsilon}\right)\right\}_{\epsilon>0}$ and $\left\{\dot{H}_{\delta(\epsilon)}\left(u^{\epsilon}\right)\right\}_{\epsilon>0}$.

By a standard argument in [4], one can get easily that

$$
\begin{gathered}
\dot{G}_{\delta(\epsilon)}\left(y^{\epsilon}\right) \rightarrow \alpha \in \partial G\left(y^{*}\right) \quad \text { weakly in } L^{2}(\Omega) \text { and } \\
\dot{H}_{\delta(\epsilon)}\left(u^{\epsilon}\right) \rightarrow \beta \in \partial H\left(u^{*}\right) \text { weakly in } L^{2}(\Omega)
\end{gathered}
$$

Next, by the hypothese $\left(H_{F}\right)$, we have $F^{\prime}\left(y^{\epsilon}\right) \rightarrow F^{\prime}\left(y^{*}\right)$ in $L\left(H_{0}^{1}(\Omega) ; Y\right)$. Thus (4.24) implies

$$
\begin{gathered}
\lambda_{0}^{\epsilon}\left[\left\langle\dot{G}_{\delta(\epsilon)}\left(y^{\epsilon}\right), z^{\epsilon}\right\rangle+\left\langle\dot{H}_{\delta(\epsilon)}\left(u^{\epsilon}\right),\left(v-u^{\epsilon}\right)^{+}\right\rangle\right]+ \\
\left\langle q^{\epsilon}, F^{\prime}\left(y^{*}\right) z-\eta+F\left(y^{*}\right)\right\rangle \geq-\theta_{\epsilon}
\end{gathered}
$$


$\forall v \in U$ and $\eta \in Q$, and $\theta_{\epsilon} \rightarrow 0$ uniformly on $v \in U$ as $\epsilon \rightarrow 0^{+}$.

Now since $F^{\prime}\left(y^{*}\right) R^{+}-Q$ is finite codimensional in $Y$, by Lemma 3.2 and (4.21), we can assume (relabelling if necessary) that $\left(\lambda_{0}^{\epsilon}, q^{\epsilon}\right) \rightarrow\left(\lambda_{0}, q\right) \neq 0$ weakly in $R \times Y^{*}$.

Thus, by taking the limits for $\epsilon \rightarrow 0$, we obtain

$$
\begin{aligned}
& \lambda_{0}\left[\langle\alpha, z\rangle+\left\langle\beta,\left(v-u^{*}\right)^{+}\right\rangle\right]+\left\langle q, F^{\prime}\left(y^{*}\right) z-\eta+F\left(y^{*}\right)\right\rangle \geq 0 \\
& \quad \text { for all } v \in U \text { and } \eta \in Q
\end{aligned}
$$

Note that $z$ depends on $v$.

After some simple calculations, we obtain

$$
0 \geq\left\langle\Psi+\lambda \beta,\left(v-u^{*}\right)^{+}\right\rangle, \quad \forall v \in U
$$

Similarly, by taking consideration of $u_{\rho}^{\epsilon}=u^{\epsilon}+\rho\left(v-u^{\epsilon}\right)^{-}$, we obtain

$$
0 \geq\left\langle\Psi+\lambda \beta,\left(v-u^{*}\right)^{-}\right\rangle, \quad \forall v \in U
$$

Thus

$$
\left\langle\Psi+\lambda \beta, v-u^{*}\right\rangle \leq 0, \quad v \in U
$$

If $(\lambda, \Psi)=0$, then by $(4.4)$, we have $\left[F^{\prime}\left(y^{*}\right)\right]^{*} q=0$. Thus in the case where $N\left[F^{\prime}\left(y^{*}\right)\right]^{*}=\{0\}$, we must have $(\lambda, \Psi) \neq 0$

This completes the proof.

\section{References}

[1] Yinbin Deng and Gengsheng Wang, Optimal Control Problem of some semilinear equations with critical exponent, J. of Nonlinear Analysis TMA, to appear.

[2] Yong J., Pontryagin maximum principle for semilinear second order elliptic partial differential equations and variational inequalities with state constraint, Diff.Int.Eqs. 5 (1992),1307-1334.

[3] Xunjing Li and Jiongmin Yong, Optimal control theory for infinite dimentional systems, Birhauser Boston 1995.

[4] V.Barbu, Analysis and Control of Nonlinear Infinite Dimensional Systems, Academic Press, New York, 1993.

[5] G.Tarantello, On Nonhomogeneous Elliptic Equations Involving Critical Sobolev's Exponent, Ann.Inst.Henri Poincare, 9(3) (1992) 281-304.

[6] Li, X., and Yong, J., Necessary Conditions of Optimal Control for Distributed Parameter Systems, SIAM J. Control Optim., 29(1991), 895-908. 